Title : will be set by the publisher

Editors : will be set by the publisher

EAS Publications Series, Vol. ?, 2013

\title{
AN UNEXPECTED RESULT FOR THE V444 CYG BINARY
}

\author{
Yaël Nazé ${ }^{1}$, Jamie Lomax ${ }^{2}$ and Jennifer Hoffman ${ }^{3}$
}

\begin{abstract}
V444 Cyg is a short-period (4d) binary composed of two massive objects, a WN star and an $\mathrm{O}$ star. The winds of the two massive components collide, generating X-ray emission. A monitoring campaign in the high-energy domain was performed using Swift and XMM-Newton, with surprising results on the collision geometry (wide shock opening angle, clear Coriolis deflection). Polarimetric data further help to understand the system properties. This new information places strong constraints on the physical parameters of the two stars.
\end{abstract}

\section{Introduction and presentation of the dataset}

Previous optical, ultraviolet and X-ray studies have suggested the presence of a wind-wind collision in the short-period binary called V444 Cyg. In 2011, Fauchez et al. (2011) reported on a partial X-ray monitoring campaign of the system: eclipses of the wind-wind collision were detected, as well as an increase of absorption when the WR star was in front. Further monitoring was needed to ascertain the system's behaviour (see Lomax et al., in preparation).

V444 Cyg has now been observed ten times by XMM-Newton. Data from 2004 (those reported in Fauchez et al. 2011) cover the first half of the orbit, while those of 2012 cover the second half of the orbit. All datasets were reduced using SAS v12.0.0. Additional Swift data were obtained in 2011 to cover the two eclipses. Polarimetric data were obtained with HPOL at the Pine Bluff Observatory between 1989 and 1994, and at the Ritter Observatory in 2012.

\section{X-ray results}

Following the same method as Fauchez et al. (2011), we progressively fitted the Xray spectrum of V444 Cyg using two absorbed thermal components with non-solar

\footnotetext{
${ }^{1}$ FNRS-Dept AGO, Allée du 6 Aout 17, B5C, University of Liège, 4000-Liège, Belgium

2 Dept of Phys. and Astr., Univ. of Oklahoma, 440 W Brooks St., Norman, OK, 73019, USA

${ }^{3}$ Dept of Phys. \& Astr., Univ. of Denver, 2112 E. Wesley Ave., Denver, CO, 80208, USA
} 
Title : will be set by the publisher

abundances. Not only the interstellar absorption, but also the temperatures and abundances of the plasma could be fixed as they appear constant, within errors, throughout the orbit.

The absorption of the hardest component strongly increases when the WR is in front of its companion, as expected in view of its dense wind. The shape of the variation, with a broad minimum, suggests a large cone opening angle (probably indicating some effect from radiative braking). Both the absorption and normalization factor of the softest component increase when the O-star is in front: this suggests that soft X-rays are generally produced far in the winds, but that additional X-rays, associated with the intrinsic soft X-ray emission of the $\mathrm{O}$-star, come into view when that star is in front.

Spectra provide detailed information on the plasma properties, but with the loss of detailed temporal characteristics. We therefore also calculated X-ray lightcurves in three energy bands (soft, hard, total). The soft lightcurve shows a highly asymmetric increase. It is partly due to absorption (as the soft X-ray flux is at its minimum when absorption is largest), but also due to the addition of the intrinsic emission of the O-star when the latter is in front (otherwise, it remains hidden by the dense WR wind) and due to Coriolis deflection (which renders the wind interaction zone asymmetric). The hard lightcurve displays two eclipses. They are due to the stars eclipsing hard X-rays produced near the line-of-centers. Note that the eclipse by the WR star is deeper, symmetric, and longer compared to that made by the O-star. The asymmetry in the secondary eclipse suggests that the emitting zone does not lie exactly on the line-of-centers (another consequence of Coriolis deflection), while the duration of both eclipses imply some eclipsing by the (inner) stellar winds in addition to the stars themselves.

\section{Polarimetric results}

HPOL enables us to study the polarization in stellar lines, not only that of the continuum. We found a significant difference in the line and continuum polarization variations, which is linked to the geometry of the system. The HeII4686 and NIV7125 lines show the same phase-locked behaviour, implying that the scattering regions are not spherically symmetric. This could happen e.g. if there is a hole in these regions and such a hole would naturally be produced in V444 Cyg as the result of the shock cone around the O-star. The observed variations suggest a large hole, i.e. a large cone opening angle, which agrees with X-ray results. However, it should be noted that preliminary modelling indicates that some scattering occurs at the shock.

\section{References}

Fauchez T., De Becker M., Nazé Y. 2011, Bul. de la Soc. Roy. des Sc. de Liège, vol. 80, 673 